$\mathrm{C}-\mathrm{A} / \mathrm{AP} / \# 284$

August 2007

\title{
Emittance growth with offset beam-beam collisions And small beam-beam parameters
}

N.P. Abreu and W. Fischer

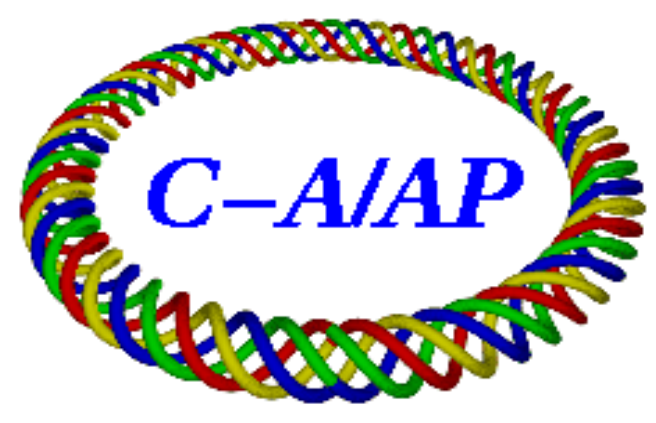

Collider-Accelerator Department Brookhaven National Laboratory

Upton, NY 11973 
BNL C-A/AP/284

\title{
Emittance growth with offset beam-beam collisions and small beam-beam parameters
}

\author{
N.P. Abreu and W. Fischer
}

August 17, 2007

\begin{abstract}
We investigate experimentally the possible enhanced emittance growth from offset beam-beam collisions. For this we displace beams at the end of a store for $15 \mathrm{~min}$, and, after removing the offset again, compare the expected luminosity with the measured one.
\end{abstract}

\section{Experiment goals and setup}

Offsets between beams under collision can cause emittance growth of both beams. This is a concern for all colliders but in particular for the LHC, where due to PACMAN effects some bunches will collide with an offset of up to $0.5 \sigma[1,2,3]$. In RHIC beams have static offsets due to uncorrected orbit errors, and modulated offsets due to mechanical triplet vibrations [4]. In simulations, these offsets lead to an increased emittance growth [5].

The experiments were performed at the end of a store when the luminosity decay can be well fitted and therefore extrapolated. The beams are then offset at the collision points for $15 \mathrm{~min}$, and then restored the "zero offset" situation. By comparing the extrapolated luminosity from the fit before the offset was introduced, with the actual luminosity after the offset was removed again, the emittance growth can be deduced. The experiments were performed during the $2004 \mathrm{Au}-\mathrm{Au}$ run and the 2004 polarized proton run.

Table 1: Summary of the offset experiments.

\begin{tabular}{|c|c|c|c|c|c|}
\hline$\overline{\operatorname{Exp} \#}$ & fillno & species & $\overline{N_{\text {bunch }}}$ & $\bar{\xi}$ & Characteristics \\
\hline 1 & 4381 & $\mathrm{Au}-\mathrm{Au}$ & $0.4 \times 10^{9}$ & $3 \times 10^{-4}$ & $\begin{array}{c}0.86 \sigma \text { horizontal offset at PHENIX and STAR } \\
\text { and } 0.87 \sigma \text { horizontal offset at BRAHMS } \\
\text { and PHOBOS for about } 15 \mathrm{~min}\end{array}$ \\
\hline 2 & 4625 & $\mathrm{Au}-\mathrm{Au}$ & $0.8 \times 10^{9}$ & $6 \times 10^{-4}$ & $\begin{array}{c}0.79 \sigma \text { vertical offset at PHENIX and STAR } \\
\text { and } 0.75 \sigma \text { vertical offset at BRAHMS } \\
\text { and PHOBOS for about } 15 \mathrm{~min}\end{array}$ \\
\hline 3 & 5259 & $\mathrm{p}-\mathrm{p}$ & $1.4 \times 10^{11}$ & $3 \times 10^{-3}$ & $\begin{array}{l}1.12 \sigma \text { horizontal in STAR and PHENIX } \\
\text { and no bump in any other IP for } 15 \mathrm{~min}\end{array}$ \\
\hline
\end{tabular}




\section{Data sets and analysis}

Tab. 1 summarizes the characteristics of each measurment. A double exponential function,

$$
f(t)=A e^{-t / \tau_{A}}+B e^{-t / \tau_{B}}
$$

was fitted to the data points before the offset and then extrapolated to the data points after the offset was removed. To compare the fits before and after the offset the spread of the data points around the fitted function was calculated, using the expression:

$$
\Delta \Sigma=\sqrt{\frac{\sum\left(y_{i}-f\left(t_{i}\right)\right)^{2}}{N-1}}
$$

and the result for each fit is shown in Tab. 2.

Table 2: Rms spread of the data points around the fitting function

\begin{tabular}{cccc}
\hline \hline Exp\# & IP & $\Delta \Sigma$ before offset & $\Delta \Sigma$ after offset \\
\hline 1 & STAR & 53 & 35 \\
& PHENIX & 53 & 40 \\
& BRAHMS & 34 & 23 \\
& PHOBOS & 33 & 27 \\
\hline 2 & STAR & 81 & 72 \\
& PHENIX & 76 & 70 \\
& BRAHMS & 50 & 56 \\
& PHOBOS & 64 & 64 \\
\hline 3 & STAR & 77 & 86 \\
& PHENIX & 74 & 70 \\
\hline \hline
\end{tabular}

In all experiments we could not measure any difference due to the offset (Figs. 1, 2 and $3)$.

\section{Summary}

We have investigated the emittance growth from transverse offset beam-beam collisions experimentally with $\mathrm{d}-\mathrm{Au}, \mathrm{Au}-\mathrm{Au}$ and p-p beams. For beam-beam parameters up to $\xi=3 \times 10^{-3}$ and 2 collisions, offsets up to $1.12 \sigma$, and offset times of 15 min we have not detected any additional transverse emittance growth.

\section{Acknowledgments}

We are thankful for discussions with W. Herr, J. Qiang, and F. Zimmermann. 

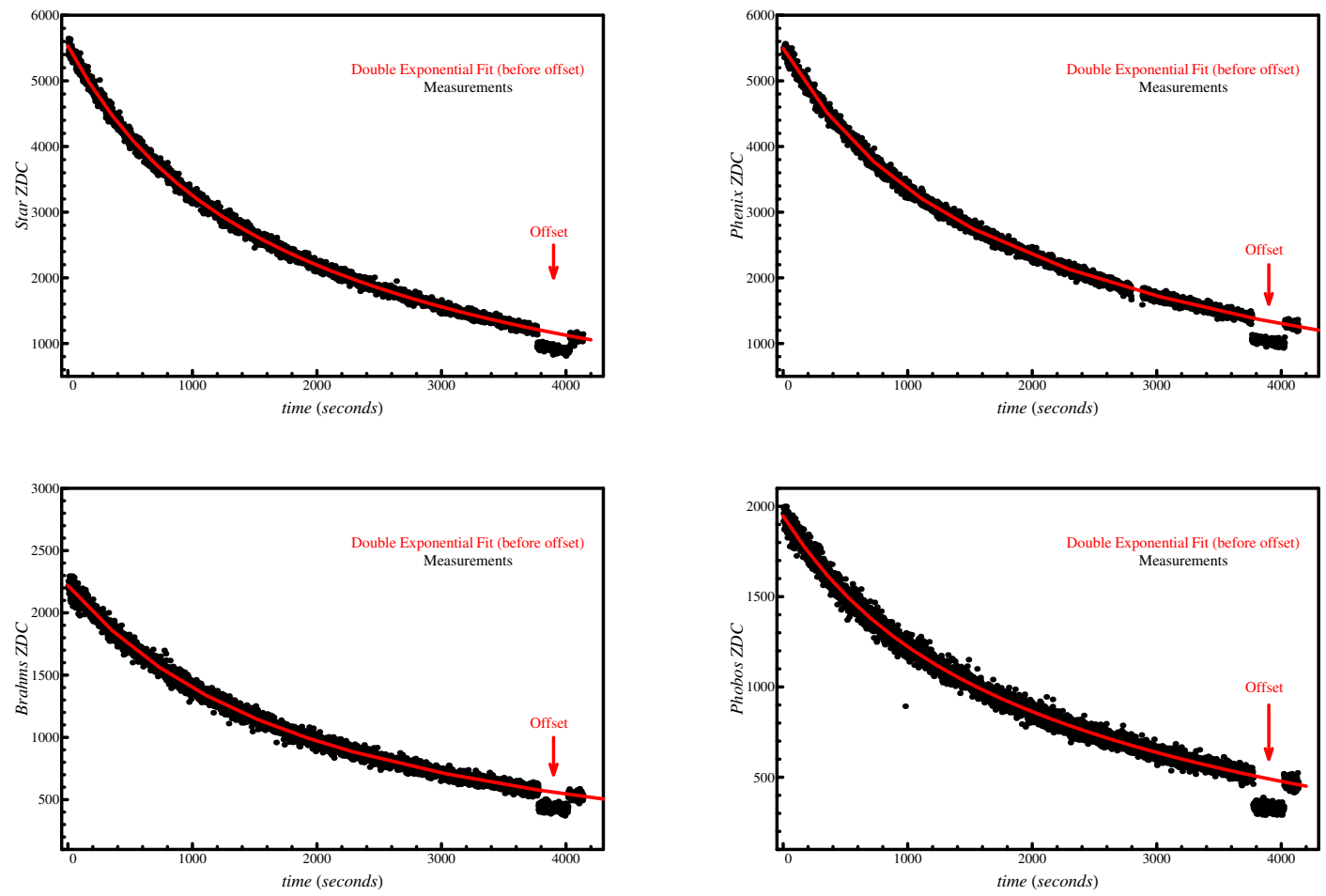

Figure 1: Luminosity measured at STAR, PHENIX, BRAHMS and PHOBOS (ZDC measurements) and the double exponential fit for experiment \# 1. 

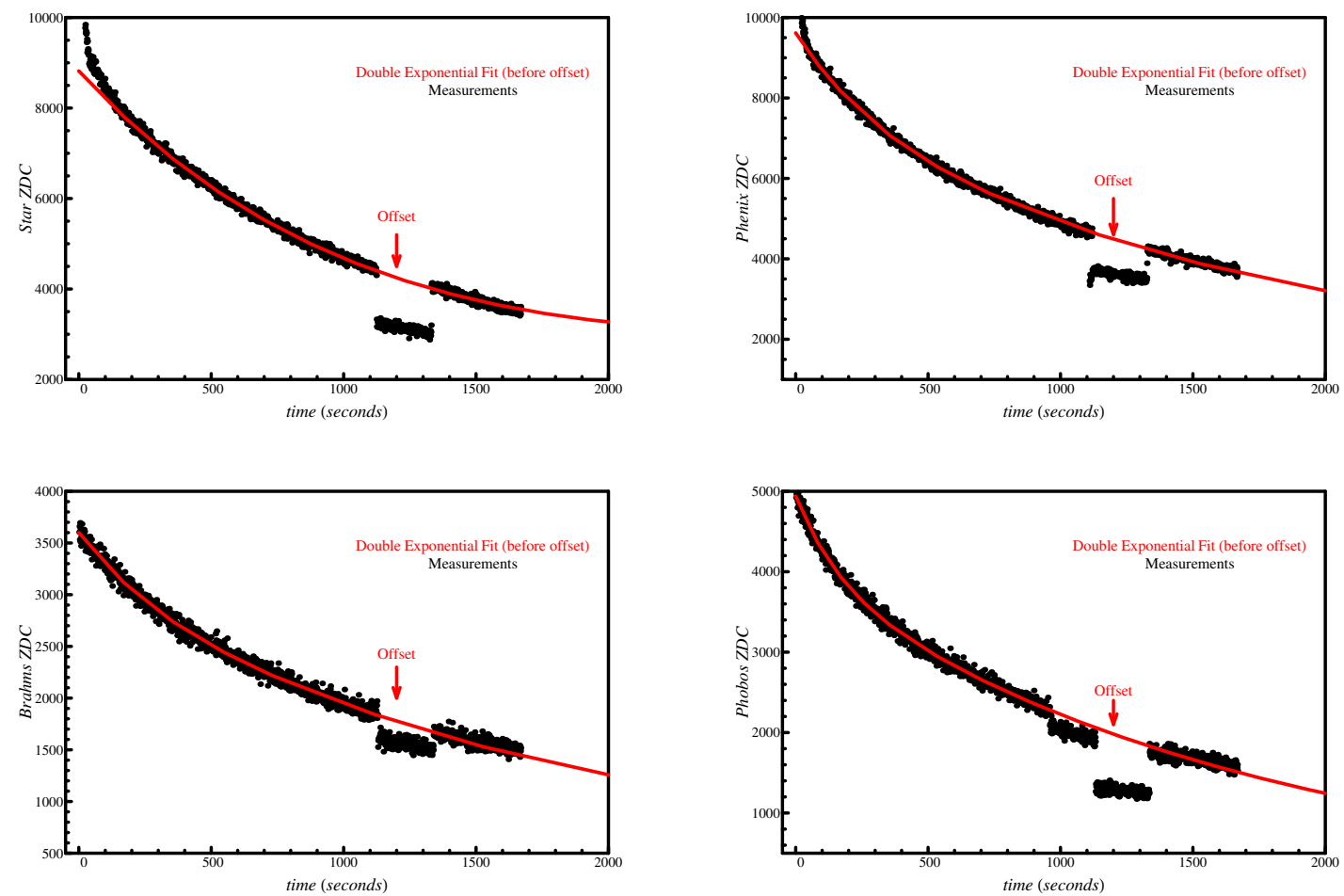

Figure 2: Luminosity measured at STAR, PHENIX, BRAHMS and PHOBOS (ZDC measurements) and the double exponential fit for experiment \# 2.
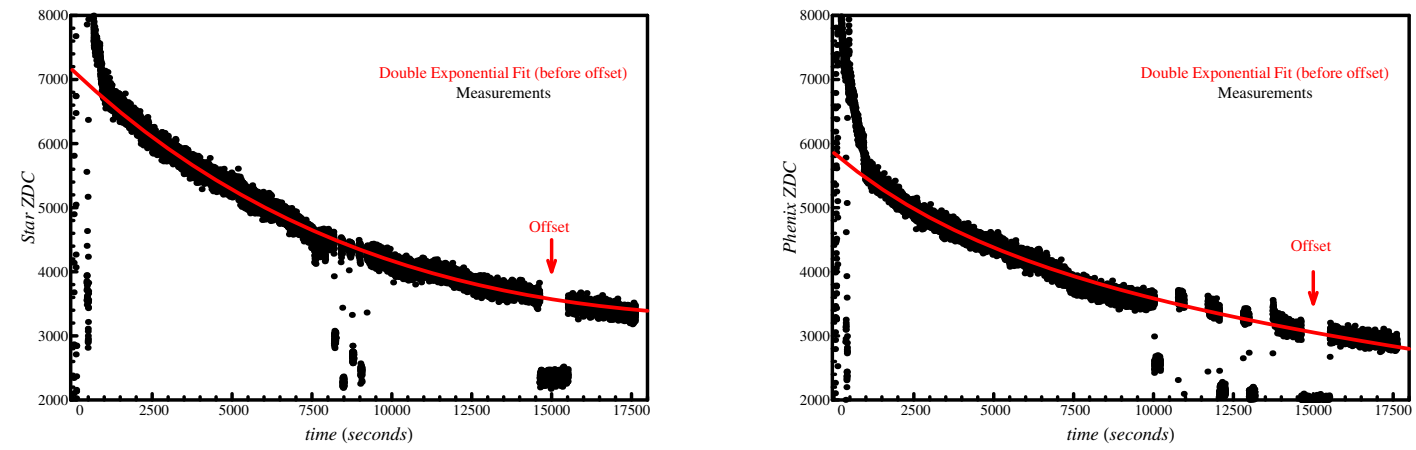

Figure 3: Luminosity measured at STAR and PHENIX (ZDC measurements) and the double exponential fit for experiment \# 3. 


\section{References}

[1] O.S. Brüning, P. Collier, P. Lebrun, S. Myers, R. Ostojic, J. Poole, P. Proudlock (editors), "LHC Design Report v.1: the LHC Main Ring", Vol. I, CERN-2004-003-V1 (2004).

[2] W. Herr, "Effects of PACMAN bunches in the LHC", CERN LHC Project Report 39 (1996).

[3] B. Muratori, "Study of offset collisions and beam adjustment in the LHC using a strong-strong simulation model", CERN LHC Project Report 593 (2002).

[4] C. Montag, R. Bonati, J.M. Brennan, J. Butler, P. Cameron, G. Ganetis, P. He, W. Hirzel, L.X. Jia, P. Koello, W. Luie, G. McIntyre, A. Nicoletti, J. Rank, T. Roser, T. Satogata, J. Schmalzle, A. Sidi-Yekhlef, J. Sondericker, and T. Tallerico, "Observation of helium flow induced beam orbit oscillations at RHIC", NIM A 564, pp. 26-31 (2006).

[5] J. Qiang, M. Furman, R.D. Ryne, W. Fischer, T. Sen, and M. Xiao, "Parallel strongstrong/weak-strong simulations of beam-beam interactions in hadron accelerators", in "Beam halo dynamics, diagnostics, and collimation", proceedings of HALO'03 and Beam-Beam'03, Montauk, New York, AIP Conference Proceedings 693 (2003). 